\title{
Alar Rim Composite Graft: A Safe and Simple Way to Correct Alar Retraction
}

Jeong Jin Chun ${ }^{1}$, Seok Min Yoon ${ }^{1}$, Syeo Young Wee ${ }^{1}$, Chang Yong Choi ${ }^{1}$, Hyuk Soo $\mathrm{Oh}^{2}$, Hyun Gyo Jeong ${ }^{1}$

${ }^{1}$ Department of Plastic and Reconstructive Surgery, Soonchunhyang Gumi Hospital, Soonchunhyang University College of Medicine, Gumi; ${ }^{2} \mathrm{~W}$ Plastic Surgery, Seoul, Korea

This work was supported in part by the Soohchunhyang University Research Fund.

No potential conflict of interest relevant to this article was reported.
Background The alar rim is a complex structure that ensures the competence of the external valves and the patency of inlets to the nasal airways. Retraction of the alar rim is caused by congenital malpositioning, hypoplasia, or surgical weakening of the lateral crura, with the potential for both functional and aesthetic ramifications. Most previously introduced procedures involved a relatively long operation time and relatively high risks of surgical complications. The purpose of this study is to introduce a novel surgical technique for alar rim connection and to present its results.

Methods After marking the extent of the correction, the recipient alar bed was created by making an incision through the vestibular skin 2-mm cephalad to the rim. Then, the composite graft was harvested from the cymba concha by removing the cartilage with its adherent anterior skin. According to the degree of retraction, the harvested composite graft was divided into 2 pieces considering the symmetry of both alar rims. The composite grafts were inserted into the defects and primary closure was done at the donor site.

Results Our surgical technique was used to correct 12 retracted alar rims in 6 patients. Caudal advancement of the alar rims was observed and the contour of the ala was corrected in all 6 patients. The mean length of follow-up was 1-year, and there were no postoperative complications, such as graft loss or disruption.

Conclusions The alar rim composite graft is a safe and simple technique for correction of short nostril and caudal transposition of the retracted alar rim.

Keywords Esthetics, Nasal cartilages, Nose, Nose deformities acquired

\section{INTRODUCTION}

The nose is a prominent feature that makes a major contribution to the aesthetics of the face [1]. Retraction or elevation (superolateral displacement) of the nostril rim can occur congenitally or iatrogenically due to scar contracture after various kinds of rhinoplasty

Received: Nov 27, 2017 Revised: May 23, 2018 Accepted: May 25, 2018 Correspondence: Hyun Gyo Jeong Department of Plastic and Reconstructive Surgery, Soonchunhyang Gumi Hospital, Soonchunhyang University College of Medicine, 179, 1 gongdan-ro, Gumi 39371, Korea.

E-mail: 33044@schmc.ac.kr

Copyright @ 2018 The Korean Society for Aesthetic Plastic Surgery.

This is an Open Access article distributed under the terms of the Creative Commons Attribution Non-Commercial License (http://creativecommons.org/licenses/by-nc/4.0/) which permits unrestricted non-commercial use, distribution, and reproduction in any medium, provided the original work is properly cited. www.e-aaps.org
[2]. Alar retraction is a deformity characterized by a distance from the alar rim to the nostril long axis exceeding $2 \mathrm{~mm}$ [3]. In this condition, the alar rim is retracted cephalad, exposing the nostril and creating an unnatural shape. Retraction of the alar rim makes the nostrils appear too big, and nasal hair is visible from the oblique and lateral views.

A defect or retraction in the nose due to trauma, tumor extirpation, or scar contracture can cause aesthetic and functional problems. Reconstruction of nasal retraction is fairly challenging for plastic surgeons.

The ala has a complex 3-dimensional (3D) shape and a convex curvature with 3 different components (inner mucosa, cartilage that supports its 3D shape, and outer skin) [4]. For these reasons, many factors must be considered when reconstructing a nose. If an incorrect reconstructive method is chosen, it becomes easy to disrupt the ala free margin by imprecise closure [5]. 
Retracted nostril rims are encountered frequently, and several surgical techniques and modifications for this condition exist; nonetheless, it is considered one of the most difficult nasal deformities to treat. To date, various procedures have been devised to correct alar retraction through composite grafts, grafting of cartilage, and soft tissue advancement. However, these procedures are complex and unpredictable, or have limited utility. Alar spreader grafts may produce a bulbous nasal tip, and lateral crural strut grafting is complicated and may result in severe postoperative edema and worsening of alar flare.

The auricular chondrocutaneous composite graft [6] is relatively commonly used for alar retraction. The helix, antihelix, concha, and lobule are often used as the donor sites [4]. The chondrocutaneous auricular composite graft has proven to be an excellent option for over 70 years because it is possible to reconstruct the structural framework, such as the cartilage, along with the inner mucosa and outer skin in a single stage, and the surgical time is shorter than that of other methods. The auricular composite graft is anatomically similar to the nostril floor because it is composed of 2 skin layers and 1 cartilage layer located between the 2 skin layers. The color and texture of auricular skin are very similar to those aspects of the nose. Auricular composite grafts rarely shrink and provide excellent support because of their similar thickness and stability. Finally, donor site morbidity is very low because the ear has a sufficient blood supply [7]. The composite graft enables straightforward coverage of the alar rim, because the ear concha has a relatively convex structure [7]. The graft can be applied to almost any part of the alar margin [2]. These advantages spurred the harvest of the chondrocutaneous composite graft from the concha.
In this study, we propose a simple procedure that effectively and safely corrects alar retraction using an auricular chondrocutaneous composite graft. This structure can effectively cover the alar rim, and has a convex curvature with different inner mucosa and outer skin layers.

\section{METHODS}

We analyzed the outcomes of 6 patients who underwent nose aesthetic operations using the chondrocutaneous composite graft. Retraction of the alar rim was the indication for the composite graft. Preoperative and postoperative photographs, outpatient charts, and hospital records were reviewed retrospectively.

\section{Donor site identification and harvest}

The composite grafts were harvested from the cymba concha by removing the cartilage with its adherent anterior skin (Fig. 1). To make the notching of the alar rim normal, we analyzed the size, location, and contour of the retracted alar rim. The cartilage harvested from the cymba concha had a horizontal length of about $15 \mathrm{~mm}$ and a vertical length of about $6 \mathrm{~mm}$. The actual size of the composite graft varied according to the degree of alar retraction (Table 1). When the alar retraction was not large, harvesting was done in only 1 ear, and the graft was divided into 2 pieces (Fig. 1). The harvested donor retaining the cartilaginous portion of the graft beyond the margins of the overlying skin which is served as stabilizing limbs was inserted into the recipient site ("inter-locking graft") [8]. Using curved Metzenbaum scissors, the skin was trimmed to expose the underlying cartilage. In this process, we made an incision or trimmed

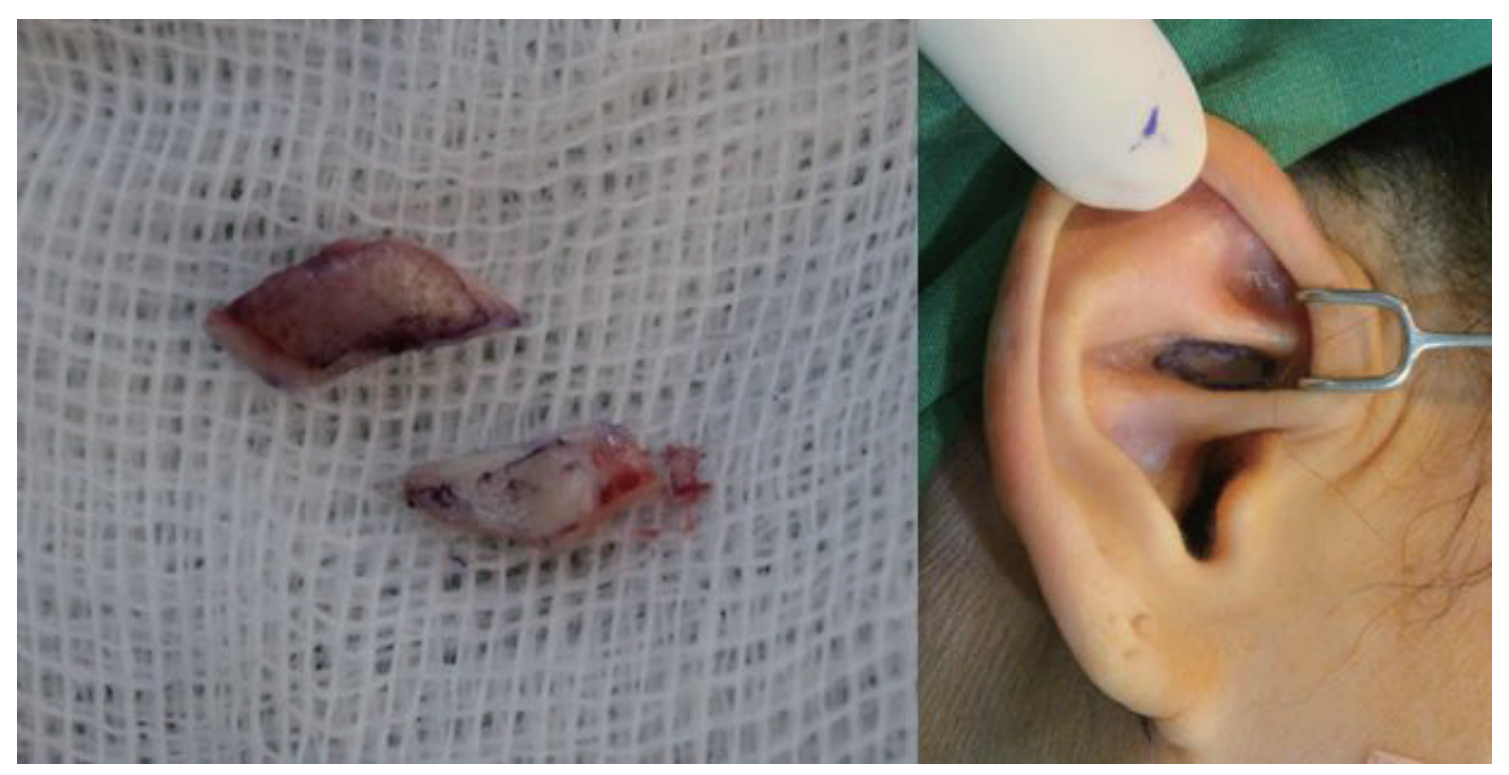

Fig. 1. Cartilage harvested from cymba concha had horizontal length of about $15 \mathrm{~mm}$ and vertical length of about $6 \mathrm{~mm}$. When the amount of alar retraction was not large, harvesting was done in only one ear and divide into two pieces. 
the skin based on the type of the alar retraction, with reference to the classification of Kim et al. [9], who classified alar retraction as medial, central, or lateral. The donor site was anesthetized with $1 \%$ lidocaine with diluted epinephrine. An incision was made at the donor site using a no. 15 blade. Harvesting the composite graft from the anterior surface of the auricular concha has been described more often than use of the posterior surface [8]. Graft tissues frequently shrink in width and thickness. To address these concerns, we designed the composite grafts to be larger than the estimated retraction. The cartilage limbs were harvested, and the cartilage extended 3 to $5 \mathrm{~mm}$ beyond the margin of the skin portion. Once ex- cised, the graft was placed in cold sterile saline-soaked gauze. The donor site was closed with simple interrupted sutures and compressive dressing was performed using a pillow.

\section{Suturing of the graft}

The incision line was drawn on the 2-mm cephalic portion of the inner alar rim of the nose, and an incision was made to secure space for the harvested cartilage (Fig. 2). At this time, the secured space was less than the size of cartilage actually harvested. The cartilage was pushed in with force and extra dissection was performed naturally in the process of inserting the cartilage (Fig. 3). The cartilage

Table 1. Preoperative and postoperative degree of alar retraction, size of the composite graft, and type of alar retraction

\begin{tabular}{|c|c|c|c|c|c|c|}
\hline \multirow{2}{*}{ Patient no. } & \multirow{2}{*}{ Alar rim } & \multicolumn{3}{|c|}{ Distance between the alar rim and the nostril long axis $(\mathrm{mm})$} & \multirow{2}{*}{$\begin{array}{l}\text { Composite graft size } \\
\text { (mm] }\end{array}$} & \multirow{2}{*}{$\begin{array}{l}\text { Type of alar } \\
\text { retraction }\end{array}$} \\
\hline & & Preoperative & Postoperative ${ }^{\text {al }}$ & Reduced distance & & \\
\hline 1 & 1 & 6.1 & 4.0 & 2.1 & $16 \times 8$ & Lateral \\
\hline \multirow[t]{2}{*}{2} & 3 & 3.0 & 2.0 & 1.0 & $17 \times 8$ & Central \\
\hline & 4 & 2.9 & 1.8 & 1.1 & & Central \\
\hline & 6 & 2.9 & 1.7 & 1.2 & & Lateral \\
\hline \multirow[t]{2}{*}{4} & 7 & 4.5 & 3.0 & 1.5 & $15 \times 6$ & Medial \\
\hline & 8 & 4.4 & 3.0 & 1.4 & & Medial \\
\hline \multirow[t]{2}{*}{5} & 9 & 3.5 & 1.7 & 1.8 & $15 \times 6$ & Lateral \\
\hline & 10 & 3.7 & 2.0 & 1.7 & & Lateral \\
\hline
\end{tabular}

a)1-year follow-up.
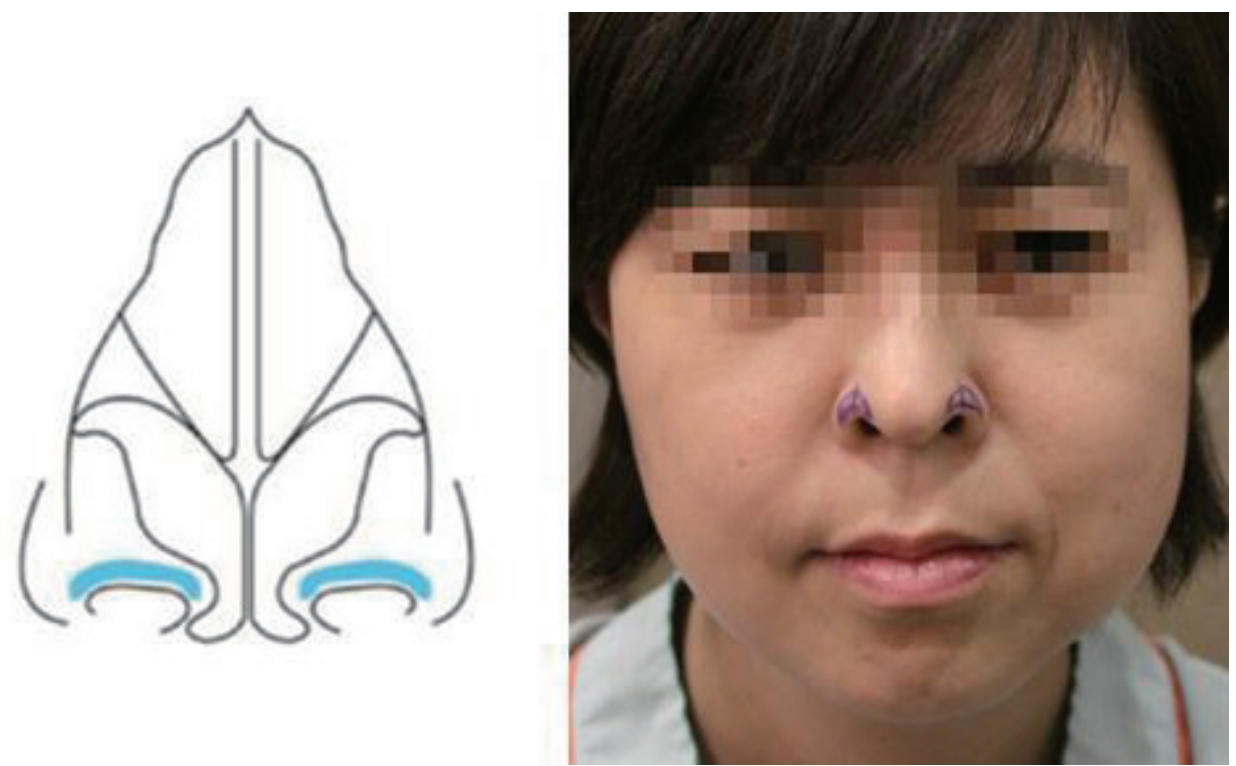

Fig. 2. We can observe the alar rim with retraction of the middle part and mark the cartilage graft insertion position by external marking. 


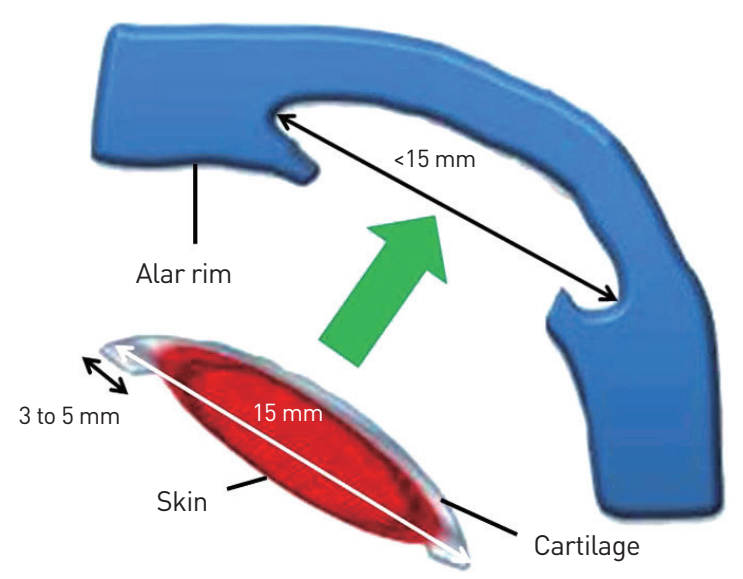

Fig. 3. Schematic drawing of alar rim composite graft. The secured space was less than the size of cartilage actually harvested. The cartilage was pushed in with force and extra dissection was performed naturally in the process of inserting the cartilage.

was tightly interlocked. An invagination technique was performed using cartilage designed to be larger than the soft tissue in order to increase the contact surface between the recipient bed and graft. When harvesting the cartilage, the perichondrium was implanted in the graft without compromising its integrity. After insertion of the cartilage, the alar rim was lengthened to the caudal area and the skin attached to the cartilage was trimmed according to the size of the skin defect. After the cartilage was interlocked, the cartilage and skin were secured using a pull-out suture (Fig. 4). This was done for the compression effect to prevent hematoma that could occur upon the forcible insertion of the cartilage. The pull-out suture also fixed the cartilage in the correct position. We applied foam to the outer skin surface during the pull-out suturing to prevent pressure sores. The inner mucosa of the graft in the vestibular area was approximated using no. 6-0 absorbable sutures. All sutures were performed using vertical mattress sutures. After suturing, a wet dressing was applied on the graft site using antibiotic ointment to improve the success rate. Immobilized compression dressing, such as Merocel $^{\circledR}$ (Medtronic Xomed, Jacksonville, FL, USA) packing, was not used.

\section{Postoperative care}

After pull-out suturing, a pillow dressing was used to maintain even pressure for 3 days. When the dressing was removed 3 days later, the graft site was swollen, with a reddish skin color. Because of the initial swelling, the suture in the vestibule was visible. When the composite graft shrunk, the suture site became invisible. Patients visited the hospital every 3 months for postoperative monitoring.

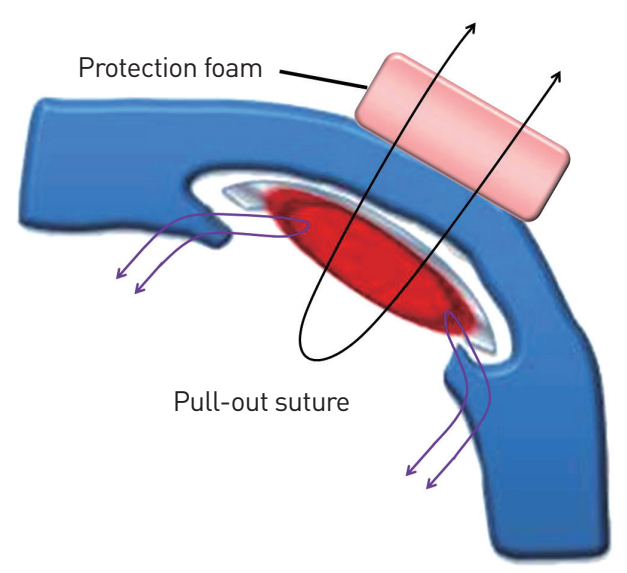

Fig. 4. Schematic drawing of alar rim composite graft. After the cartilage was interlocked, the cartilage and skin were secured using a pull-out suture (black arrow). Protection foam was applied to the outer skin surface during the pull-out suturing to prevent pressure sores. The inner mucosa of the graft in the vestibular area was approximated using no. 6-0 absorbable sutures (purple arrows). All sutures were conducted by vertical mattress suture.

\section{RESULTS}

Over a 2-year period, 6 patients underwent the operation using the chondrocutaneous composite graft. The 11 alar rim composite grafts survived completely and no patients showed a noticeable contour deformity on the donor site. We achieved satisfactory results in the correction of retracted alar rims. Caudal advancement of the alar rims was observed. The mean value of the distance between the alar rim and the nostril long axis was $3.8 \mathrm{~mm}$ preoperatively and $2.4 \mathrm{~mm}$ postoperatively. The average reduction in the distance was $1.5 \mathrm{~mm}$. Various types of alar retraction were observed (Table 1). The nasal framework and contour were acceptably maintained. After a 1-year follow-up, all composite grafts had shrunk. Shrinkage was minimized by the use of cartilage with a 3 to $5 \mathrm{~mm}$ extension in comparison to the skin. At the 1-year follow-up, there were no postoperative complications, including graft loss or disruption.

\section{Case 1}

A 43-year-old woman was referred to our plastic surgery unit for correction of a retracted alar rim. The patient's chief complaint was that the nasal cavity could be seen from the front, and she exhibited the lateral type of alar retraction. She had no previous specific trauma history and retraction was observed bilaterally. The donor site was planned to target the cymba concha of the ipsilateral ear. A combined skin and free composite cartilage graft measuring $15 \times 6 \mathrm{~mm}$ was harvested from the anterior surface of the cymba concha, considering the lateral type of alar retraction. After meticulous dissection of the graft recipient site, we inserted the cartilage and sutured 


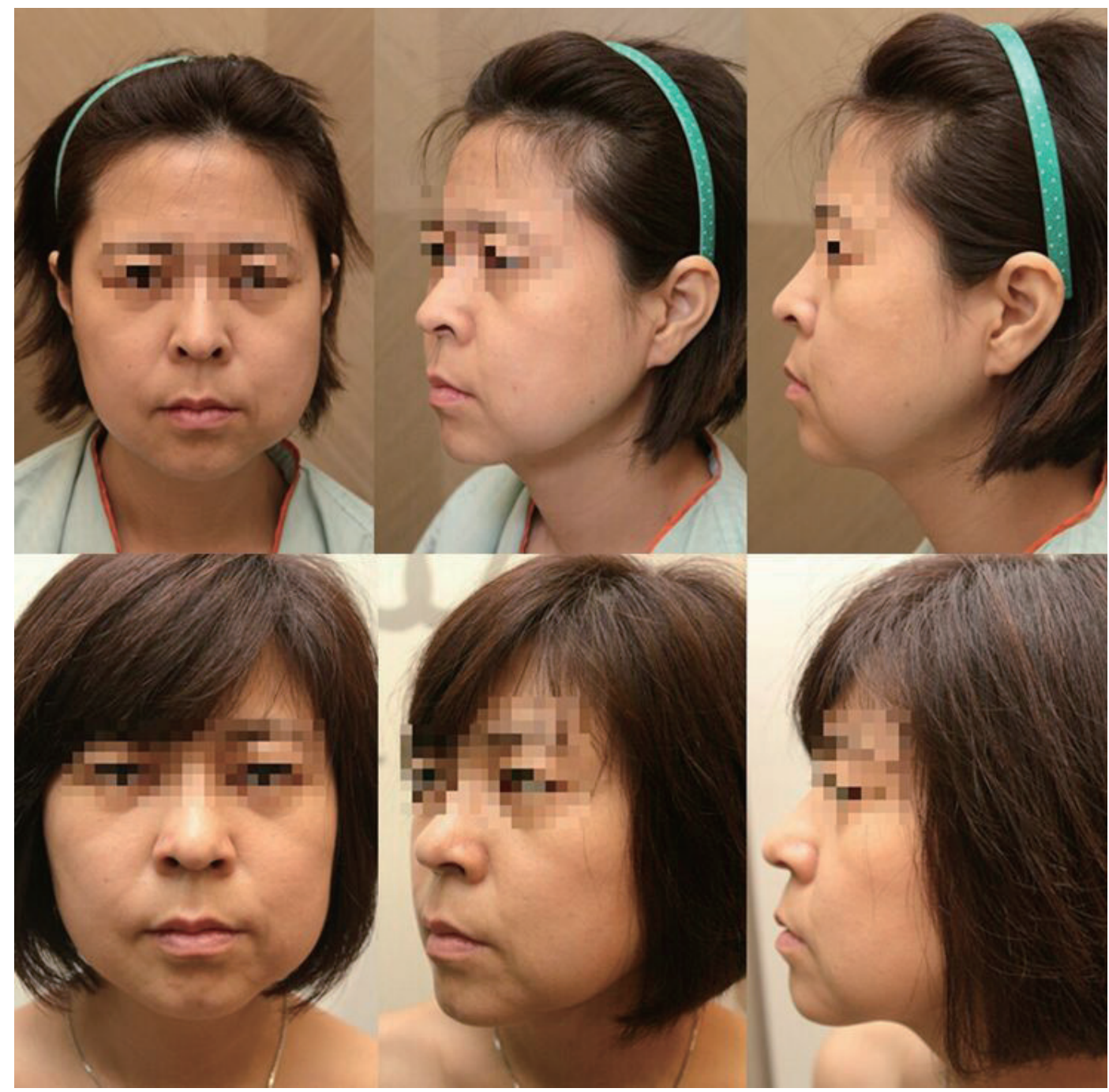

Fig. 5. The above three photographs are preoperative images. The below three photographs are postoperative images. The vestibule is exposed due to alar retraction and the lateral type of alar retraction is observed. The alar contour is maintained after one year of composite graft.

the composite graft at the nostril lining. The graft stabilized without any postoperative complications or problems. At 1-year, the alar contour was maintained, with good aesthetic and functional outcomes. The nasal cavity was relatively concealed in the frontal view (Fig. 5).

\section{Case 2}

A 48-year-old man presented with a unilateral retracted nostril rim. His preoperative frontal appearance showed a notch-like configuration in the superior region of the nostril rim. We harvested an auricular chondrocutaneous composite graft as in case 1, and inserted the composite graft. After the operation, the nostril asymmetry was corrected and an acceptable range of contour deformity was present on the outer side of the nostril. There were no complications and the patient was satisfied with the surgical outcome. At the 1-year follow-up, the retraction that existed preoperatively was corrected. The area in which the vestibule was visible within the external valve was reduced (Fig. 6).

\section{DISCUSSION}

Because the alar rim plays a crucial role in the balance of the nasal base, deformities of the alar rim cause nostril exposure and an undesirable nasal shape. Thus, for an aesthetically pleasing nose, an ideal alar-columellar relationship must be obtained. Alar retraction refers to an alar rim greater than $2 \mathrm{~mm}$ and a distance from the long axis of the nostril to the columella of 1 to $2 \mathrm{~mm}$ [3]. Meyer and Kesselring [10] introduced a method for lowering the alar rim with a graft made of a strip of alar cartilage. Ellenbogen [11] modified this method to create a combined technique with a local skin 


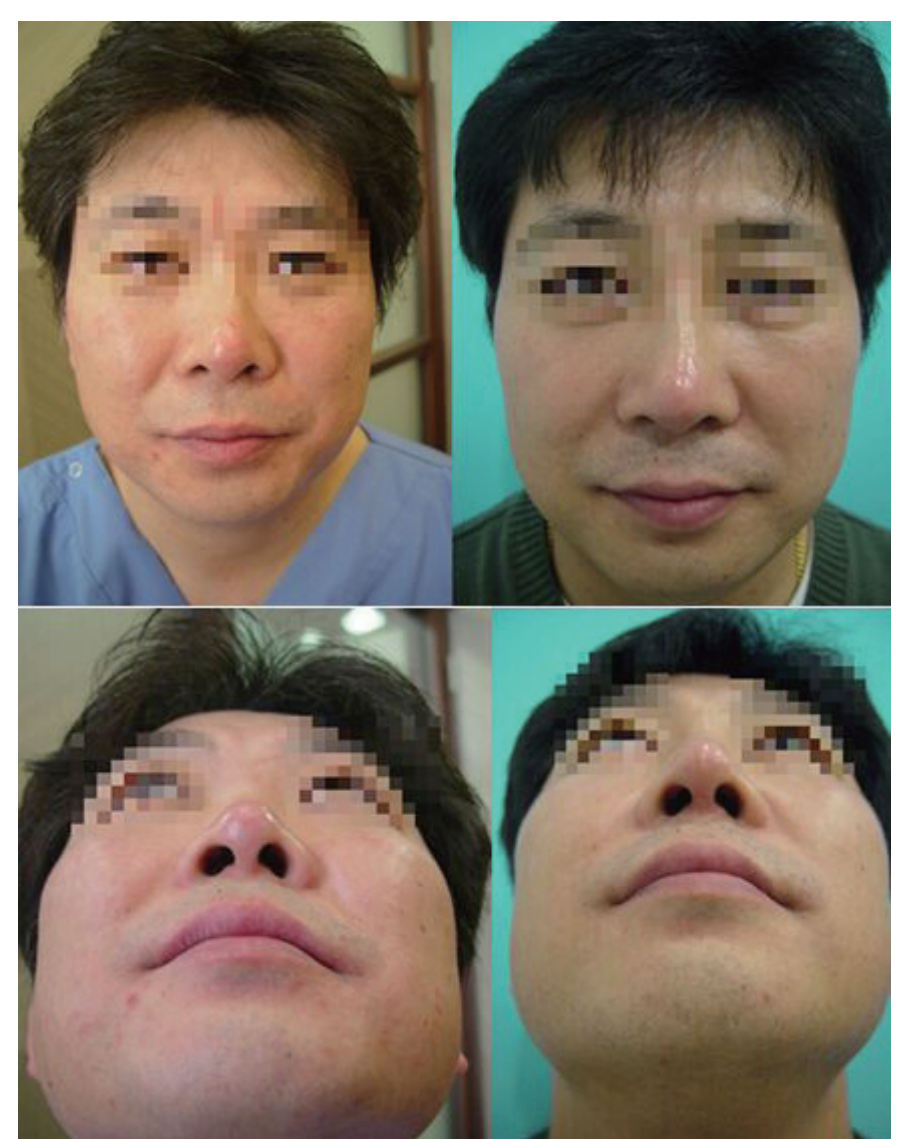

Fig. 6. One year after the operation, the retraction seen in the preoperative photo was corrected. It can be seen that the area in which the vestibule is visible within the external valve is reduced. In the worm's eye view, you can see that the notched alar is straightened.

flap and a cartilage graft (septal, lower lateral, or auricular). The modification leaves a raw surface on the internal mucosa, which could lead to postoperative complications including distortion, retraction, and protrusion of the grafted cartilage. Guyuron [12] used internal V-to-Y advancement with or without a cartilage graft for severe alar retraction. Rohrich et al. [13] utilized alar contour grafting with septal cartilage. In patients with mild to moderate alar retraction, alar contour grafts are effective. In moderate to severe cases, alar spreader grafts, lateral crural strut grafts, and alar extension grafts are useful. Composite grafting is indicated for severe alar retraction.

Compared to the previous technique, it is preferable to use conchal cartilage, since the intrinsic curvature of the conchal cartilage resembles that of the lateral crus of the lower lateral cartilage. The composite graft has a flexible structure and can be used to transfer small components. Moreover, composite graft procedures are suitable for Koreans and others with similar narrow alar and columellar proportions, which need a complex and delicate operation technique. However, all grafts undergo some degree of shrinkage. In particular, shrinkage of a composite graft on the nose can be aesthetically objectionable. To prevent shrinkage, a modified composite graft was devised, in which the auricular cartilage is 2 to $3 \mathrm{~mm}$ wider than the auricular skin border [1]. This enables the easy insertion of the cartilage into the nostril recipient tissue and can strengthen the nasal framework, which minimizes the scar contracture that may occur after graft shrinkage [1].

In order to stabilize the graft, Hirohi and Yoshimura [14] combined auricular composite grafts and postoperative anchoring suspension with a nasal retainer and external plastic cast. However, the nasal retainer in the nostril and the external plastic cast can cause discomfort to patients. In our surgical technique, there is no need for an inner retainer to hold the nostril in place or a device for compression, thanks to the 2 cartilage limbs with a solid character. This is quite comfortable for the patient. The cartilage limbs are useful for strengthening the nasal framework, minimizing scar contracture that may occur in the future, and maintaining the nostril shape. Moreover, we fixed the cartilage and vestibular mucosa cephalic to the marginal incision with a 1-point pull-out suture for additional stability.

In our procedure, the composite graft was harvested from the anterior aspect of the auricle. This is because the volume of the skin cover and subcutaneous soft tissue of the posterior surface are thicker than that of the anterior surface. This reduced the morbidity of the donor site. Furthermore, if the degree of retraction was not large, we divided the cartilage into 2 pieces to minimize morbidity at the donor site. In addition, when harvesting the composite graft, it is important to select an area of the auricle that closely resembles the curved configuration of the nostril rim. Thus, we used the anterior wall of the concha.

From the point of view of harvesting of the graft, we considered the type of alar retraction. According to Kim et al. [9], alar retraction can be classified as medial, central, or lateral. We made an incision or trimmed the skin paddle based on the type of the alar retraction, with reference to the classification of Kim et al. [9]. Doing so yielded better aesthetic outcomes of the postoperative shape of the alar rim. For example, the alar retraction of the patient in case 1 was classified as the lateral type. We made an incision to make the lateral portion larger so that the lateral retraction could be corrected. All suturing of the graft was performed using vertical mattress sutures. This caused eversion of the wound margin, allowing the final surface to be wider. This in turn enabled a wider correction of the alar retraction. Our results indicated that this procedure could correct not only the degree of alar retraction, but also the shape of the alar rim.

There are several potential limitations of this study. The biggest limitation is that of size. Large composite grafts are infrequently used because a larger size reduces the survival rate of the graft [1]. Composite grafts require more metabolites than simple skin grafts and must undergo rapid revascularization to be successful [15]. 
The revascularization of a composite graft starts in the subdermal plexus of the graft and recipient donor bed [15]. However, this circulation is limited in terms of its ability to extend beyond the margin of the composite graft [15]. Furthermore, the survival of the cartilage of the composite graft depends on the passive diffusion of oxygen and nutrients into the perichondrium from around the well-vascularized tissue [15]. Therefore, vascularity is more important in composite grafts, so the restriction of length is more absolute [15]. Second, the auricular cartilage is vulnerable to postoperative infection [5]. In fact, prophylactic antibiotics were used to prevent infection of the recipient cartilage and donor suture site. In addition, the postoperative follow-up period was relatively short. Postsurgical monitoring for $>1$ year is advisable to check for relapses of alar retraction.

\section{CONCLUSION}

The auricular conchal chondrocutaneous composite graft is a reliable option for correction of a retracted alar rim. Although additional cases should be analyzed, this is a more simple and safe method than other procedures in that it involves harvesting a lesser volume of cartilage and a shorter operation time. Using this procedure, reconstruction in cases of alar retraction is possible. As a result, patient satisfaction can be obtained without donor site morbidity.

\section{PATIENT CONSENT}

Patients provided written consent for the use of their images.

\section{REFERENCES}

1. Ferreira S, Ayres Quaresma LE, Timóteo CA, et al. The primary closure approach of dog bite injuries of the nose. J Craniofac Surg 2014; 25:e216-8.
2. Peach AH, Stretch JR. Improved selection and management of the auricular composite graft donor site. Br J Plast Surg 2004;57:797-9.

3. Gunter JP, Rohrich RJ, Friedman RM. Classification and correction of alar-columellar discrepancies in rhinoplasty. Plast Reconstr Surg 1996; 97:643-8.

4. Son D, Kwak M, Yun S, et al. Large auricular chondrocutaneous composite graft for nasal alar and columellar reconstruction. Arch Plast Surg 2012;39:323-8.

5. Klinger M, Maione L, Villani F, et al. Reconstruction of a full-thickness alar wound using an auricular conchal composite graft. Can J Plast Surg 2010;18:149-51.

6. Brown JB, Cannon B. Composite free grafts of two surfaces of skin and cartilage from the ear. Ann Surg 1946;124:1101-7.

7. Haug MD, Rieger UM, Witt $P$, et al. Managing the ear as a donor site for composite graft in nasal reconstruction: update on technical refinements and donor site morbidity in 110 cases. Ann Plast Surg 2009; 63:171-5.

8. Ratner D, Katz A, Grande DJ. An interlocking auricular composite graft. Dermatol Surg 1995;21:789-92.

9. Kim JH, Park SW, Oh WS, et al. New classification for correction of alar retraction using the alar spreader graft. Aesthetic Plast Surg 2012; 36:832-41.

10. Meyer R, Kesselring UK. Sculpturing and reconstructive procedures in aesthetic and functional rhinoplasty. Clin Plast Surg 1977;4:15-39.

11. Ellenbogen R. Alar rim lowering. Plast Reconstr Surg 1987;79:50-7.

12. Guyuron B. Alar rim deformities. Plast Reconstr Surg 2001;107:85663.

13. Rohrich RJ, Raniere J Jr, Ha RY. The alar contour graft: correction and prevention of alar rim deformities in rhinoplasty. Plast Reconstr Surg 2002;109:2495-505; discussion 506-8.

14. Hirohi T, Yoshimura K. Surgical correction of retracted nostril rim with auricular composite grafts and anchoring suspention. Aesthetic Plast Surg 2003;27:418-22.

15. Adams C, Ratner D. Composite and free cartilage grafting. Dermatol Clin 2005;23:129-40, vii. 